Bull. Mater. Sci., Vol. 6, No. 1, February 1984, pp. 2I-25.

(C) Printed in India.

\title{
Magnetic properties of metallic glass $\mathrm{Fe}_{39} \mathrm{Ni}_{39} \mathrm{Mo}_{4} \mathrm{Si}_{6} \mathrm{~B}_{12}$
}

\author{
B BHANU PRASAD, ANIL K BHATNAGAR, \\ $S$ VENKATARAMAN and M N CHANDRASEKHARAIAH*
}

School of Physics, University of Hyderabad, Hyderabad 500134 , India

* Bharat Heavy Electricals Limited (R \& D), Hyderabad 500539, India

\begin{abstract}
Magnetic metallic glass $\mathrm{Fe}_{39} \mathrm{Ni}_{39} \mathrm{Mo}_{4} \mathrm{Si}_{6} \mathrm{~B}_{12}$ has been studied by the ${ }^{57} \mathrm{Fe}$ Mössbauer spectroscopy. Mössbauer spectra consist of broad and overlapping six-line pattern below the Curie temperature. The Curie and crystallization temperatures of this metallic glass have been determined to be $575 \pm 3 \mathrm{~K}$ and $725 \pm 3 \mathrm{~K}$, respectively. The hyperfine magnetic field at room temperature is approximately $225 \mathrm{kOe}$. The reduced hyperfine fields of this sample decrease much faster than that observed for other iron-rich metallic glasses like $\mathrm{Fe}_{40} \mathrm{Ni}_{40} \mathrm{~B}_{20}, \mathrm{Fe}_{80} \mathrm{~B}_{20}$ etc. This behaviour is attributed to the presence of molybdenum atoms in the sample.
\end{abstract}

Keywords. Metallic glasses; Mössbauer spectroscopy; magnetic properties.

\section{Introduction}

Magnetic metallic glasses, produced by the rapid quenching of a melt of an iron-rich alloy, have become technological important materials. These materials have high saturation induction and other excellent soft magnetic properties which are now presently being exploited for various applications in electromagnetic industry (Luborsky et al 1978). The magnetic properties of these glasses not only depend upon the alloy composition but also on the details of the preparation method, i.e. quenching rate etc. Therefore, the investigation of the magnetic properties of commercially available metallic glasses is of importance. In this paper we report the results of the Mössbauer investigations of "as-received" metallic glass $\mathrm{Fe}_{39} \mathrm{Ni}_{39} \mathrm{Mo}_{4} \mathrm{Si}_{6} \mathrm{~B}_{12}$ and is a part of our extensive investigations of magnetic and electrical properties of magnetic metallic glasses (Bhamu Prasad et al 1980, 1981a,b,c; 1983; Bhatnagar et al 1982; Bhatnagar and Ravi 1983).

\section{Experimental}

Metallic glass $\mathrm{Fe}_{39} \mathrm{Ni}_{39} \mathrm{Mo}_{4} \mathrm{Si}_{6} \mathrm{~B}_{12}$ was obtained from Vacuumschmelze (West Germany) in a thin ribbon form having a width of approximately $1 \mathrm{~cm}$ and a thickness of approximately $60 \mu \mathrm{m}$. The Mössbauer absorber was prepared using two widths of the ribbon clamped between two thin annular rings of oxygen free copper. Mössbauer spectra were recorded on an Elscint spectrometer coupled with 256 channel MCA. The standard transmission geometry was used and the spectrometer in the constant acceleration mode was used. The source was $25 \mathrm{mCi} \mathrm{Co}$ in rhodium matrix. The constant Doppler velocity thermal-scan method was used to determine Curie and crystallization temperatures accurately (Chien and Hasegawa 1977). 


\section{Results and discussion}

Mössbauer spectra of "as-received" sample were recorded in the temperature range $77-900 \mathrm{~K}$. Figure 1 shows the spectra recorded at 297,600 and $750 \mathrm{~K}$. The room temperature ( $297 \mathrm{~K}$ ) spectrum consists of broad and overlapping but well-resolved six absorption lines which are characteristic of the ferromagnetic state of the sample. The average linewidth of the outer lines is approximately $1.44 \mathrm{~mm} / \mathrm{sec}$, about six times larger than the natural line width of iron. Similar spectra have been observed at other temperatures but below $575 \mathrm{~K}$. These broad and overlapping lines in the spectra are due to the amorphous nature of the sample. Random atomic arrangement in an amorphous solid gives rise to a large number of inequivalent iron-sites. This results in a number of overlapping spectra characteristic of each inequivalent iron-site, and hence the broadening of absorption lines in the spectra. The spectra at and above $575 \mathrm{~K}$ but below $725 \mathrm{~K}$ consist of quadrupole-split doublet in each spectrum. Figure $1 \mathrm{~b}$ shows the quadrupole-split spectra at $600 \mathrm{~K}$. The splitting between the doublet is approximately $0.47 \mathrm{~mm} / \mathrm{sec}$ at this temperature. A slight asymmetry in the doublet should also be noticed which probably arises due to a distribution of isomer-shifts in the amorphous sample. A complex spectrum was observed at $725 \mathrm{~K}$ indicating the onset of crystallization at this temperature. Mössbauer spectra taken at $750 \mathrm{~K}$ is shown in figure 1c in which extra absorption lines besides the quadrupole-split doublet clearly show the precipitation of $\alpha-\mathrm{Fe}$. From these observations it is concluded that the Curie and crystallization temperatures of "as-received" $\mathrm{Fe}_{39} \mathrm{Ni}_{39} \mathrm{Mo}_{4} \mathrm{Si}_{6} \mathrm{~B}_{12}$ sample are approximately 575 and $725 \mathrm{~K}$, respectively. An independent check of these characteristic temperatures of the amorphous sample was done by the constant Doppler velocity

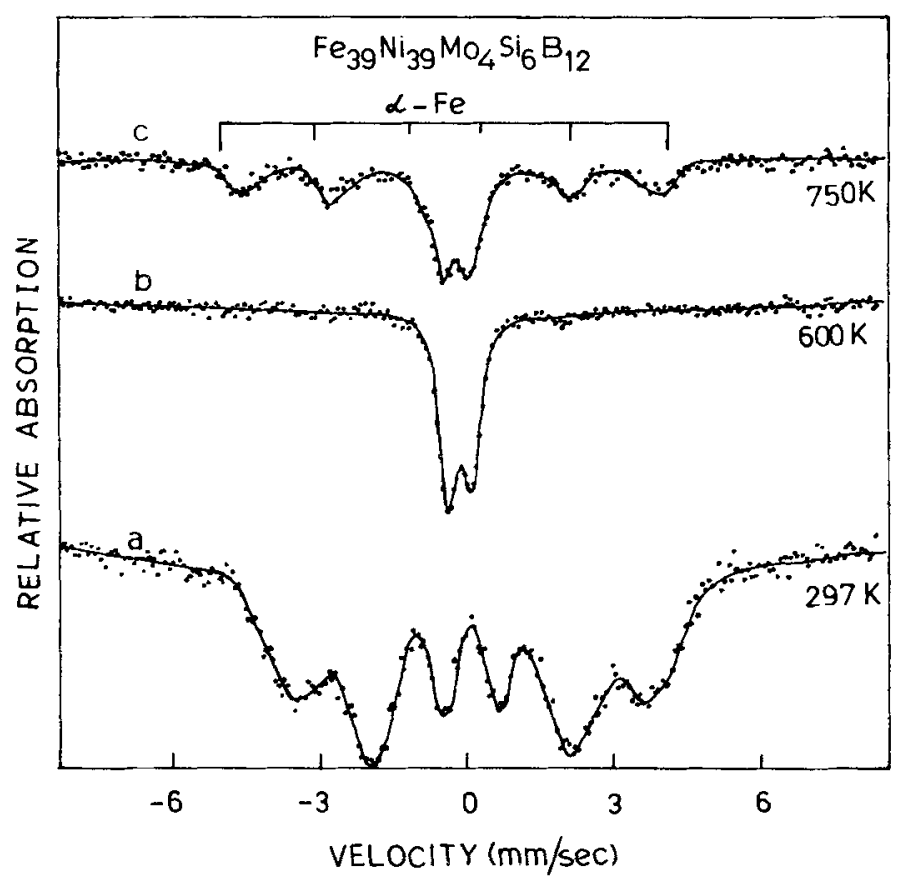

Figure 1. Mössbauer spectra of $\mathrm{Fe}_{39} \mathrm{Ni}_{39} \mathrm{Mo}_{4} \mathrm{Si}_{6} \mathrm{~B}_{12}$. 
thermal-scan method which also established the Curie and crystallization temperatures to be $575 \pm 3 \mathrm{~K}$ and $725 \pm 3 \mathrm{~K}$, respectively, in agreement with previous results.

The temperature dependence of the hyperfine or internal magnetic field $H_{\text {eff }}(T)$, as determined from the splitting of the outer-most absorption lines in the spectrum, is shown in figure 2. The extrapolation of the $H_{\text {eff }}(T)$ vs $T$ curve to $T \rightarrow 0$ and $H_{\text {eff }}(T) \rightarrow 0$ gives $H_{\text {eff }}(0)=278 \pm 3 \mathrm{kOe}$ and the Curie temperature $T_{c}=575 \pm 5 \mathrm{~K}$, respectively. The value of $T_{c}$, deduced by this method, is also in agreement with the thermal-scan method.

The reduced hyperfine magnetic fields, $H_{\mathrm{eff}}(T) / H_{\mathrm{eff}}(0) v s$ the reduced temperature, $T / T_{\text {c }}$, are plotted in figure 3 along with the Brillouin curve for spin 1 which represents the approximate behaviour of elemental ferromagnets like $\mathrm{Fe}$. It is noticed that the experimental points for $\mathrm{Fe}_{39} \mathrm{Ni}_{39} \mathrm{Mo}_{4} \mathrm{Si}_{6} \mathrm{~B}_{12}$ lie much below the Brillouin curve indicating that $H_{\text {eff }}(T)$ decreases much faster with increasing temperature when compared with elemental ferromagnets. This behaviour has been observed in other metallic glasses (Chien and Hasegawa 1977; Bhanu Prasad et al 1980; Bhatnagar et al

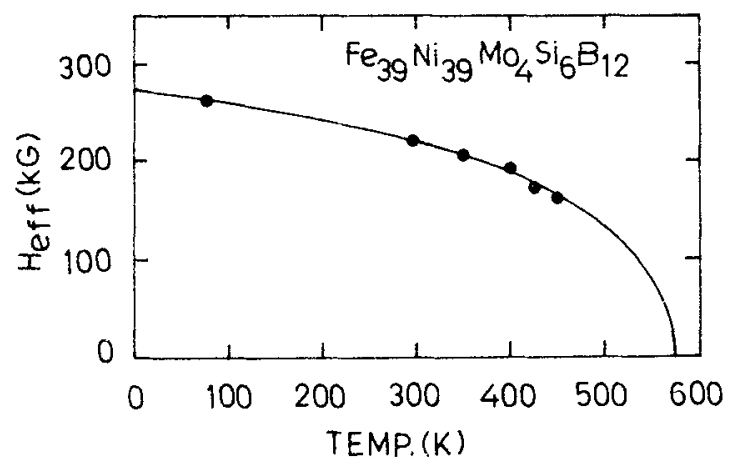

Figure 2. Temperature dependence of hyperfine magnetic field $H_{\text {eff }}(T)$ of $\mathrm{Fe}_{39} \mathrm{Ni}_{39} \mathrm{Mo}_{4} \mathrm{Si}_{6} \mathrm{~B}_{12}$ in the glassy state.

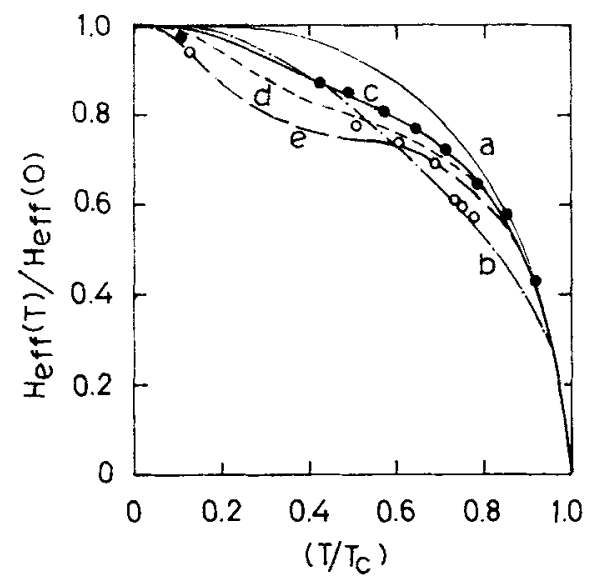

Figure 3. Reduced hyperfine magnetic field $v s$ reduced temperature. Circles represent experimental points for $\mathrm{Fe}_{39} \mathrm{Ni}_{39} \mathrm{Mo}_{4} \mathrm{Si}_{6} \mathrm{~B}_{12}$ and solid spheres represent experimental points for $\mathrm{Fe}_{40} \mathrm{Ni}_{40} \mathrm{~B}_{20}$. Curves a, b, c, d, and e are obtained from equation (1) for $\delta=0, \delta=0.5, \delta$ $=0.6\left(1-t^{2}\right), \delta=0.7\left(1-t^{2}\right)$ and $\delta=0.8\left(1-t^{2}\right)$, respectively, where $t=T / T_{c}$. 
1982; Saegusa and Morrish 1982). This behaviour of amorphous ferromagnets is usually explained, at least qualitatively, on the basis of Handrich's model (Handrich 1969 ) in which fluctuations in the exchange interactions due to the random atomic arrangement in an amorphous solid account for this behaviour of $H_{\mathrm{eff}}(T)$. In this model the reduced hyperfine magnetic field can be expressed as

$$
h=H_{\mathrm{eff}}(T) / H_{\mathrm{eff}}(0)=\frac{1}{2} B_{s}[(1+\delta) x]+\frac{1}{2} B_{s}[(1-\delta) x]
$$

where $x=[3 S /(S+1)](h / t)$ with $t=T / T_{c}, B_{s}$ is the Brillouin function for spin $S$, and $\delta(0<\delta<1)$ is a measure of fluctuations in the exchange interactions. For $\delta=0$, (1) reduces to the form $h=B_{s}(x)$ applicable to crystalline elemental ferromagnets. In figure 3 we have also drawn a curve using (1) in which $\delta=0.5$ and $S=1$ have been used. It is observed that the agreement between experimental points and this curve is good close to $T_{c}$, but not good at low temperatures. It has been previously shown by us that the agreement between experimental points and (1) can be improved vastly if $\delta$ is assumed to be a temperature-dependent parameter, i.e. $\delta=\delta_{0}\left(1-t^{2}\right)$ in which $\delta_{0}$ is used as a fitting parameter. It is found that for a good fit the value of $\delta_{0}$ lies between 0.6 and 0.7 (Bhanu Prasad et al 1980; Bhatnagar et al 1982). For example, we have shown that data for $\mathrm{Fe}_{40} \mathrm{Ni}_{40} \mathrm{~B}_{20}$ show a good agreement with (1) if $\delta=0.6\left(1-t^{2}\right)$ and $S=1$ are used in the equation. However, we find that a similar agreement is not found for $\mathrm{Fe}_{39} \mathrm{Ni}_{39} \mathrm{Mo}_{4} \mathrm{Si}_{6} \mathrm{~B}_{12}$ and the experimental points lie much below the curve for $\delta=0 \cdot 7\left(1-t^{2}\right)$ although some points fall on the curve for $\delta=0.8\left(1-t^{2}\right)$ as shown in the figure. We believe that this deviation may be due to the presence of molybdenum atoms in this sample. Further detailed investigation is in progress and will be reported at a later date.

\section{Conclusions}

Mössbauer spectroscopy has been used to study metallic glass $\mathrm{Fe}_{39} \mathrm{Ni}_{39} \mathrm{Mo}_{4} \mathrm{Si}_{6} \mathrm{~B}_{12}$ in the temperature range 77-900 K. The observed Mössbauer spectra are typical of those of other iron-rich metallic glasses exhibiting broad and overlapping but well resolved six-line spectra below Curie temperature. The Curie and crystallization temperatures of this sample have been found to be $575 \pm 3 \mathrm{~K}$ and $725 \pm 3 \mathrm{~K}$, respectively. The hyperfine or internal magnetic field, $H_{\text {eff }}(T)$, decreases much faster with rise in temperature than predicted by the Brillouin curve for spin one, as well as in comparison with other metallic glasses which contain only ferromagnetic transition metals and metalloids, i.e., $\mathrm{Fe}-\mathrm{Ni}-\mathrm{B}, \mathrm{Fe}-\mathrm{B}-\mathrm{Si}$ etc.

\section{Acknowledgements}

AKB thanks the Science and Engineering Research Council (DST, India) and the Department of Atomic Energy, India for the support of this work. sv thanks CSIR, India for the fellowship.

\section{References}

Bhanu Prasad B, Bhatnagar A K and Jagannathan R 1980 Solid State Commun. 36661 Bhanu Prasad B and Bhatnagar A K 1981a Bull. Mater. Sci. 319 
Bhanu Prasad B, Bhatnagar A K and Jagannathan R 1981b Proc. of Silver Jubilee Physics Symposium, Department of Atomic Energy, Bombay, India C24 97

Bhanu Prasad B, Bhatnagar A K and Jagannathan R 1981c Proceedings of International Conference on Applications of the Mössbauer Effect, Jaipur, India (To be published)

Bhanu Prasad B, Bhatnagar A K and Jagannathan R 1983 J. Appl. Phys. 542019

Bhatnagar A K, Bhanu Prasad B, Ravi N, Jagannathan R and Anantharaman T R 1982 Solid State Commun. 44905

Bhatnagar A K and Ravi N 1983 Phys. Rev. B28 359

Chien C L and Hasegawa R 1977 Phys. Rev. B16 3024

Handrich K 1969 Phys. Status. Solidi. 32 K55

Luborsky F E, Becker J J, Frischmann P G and Johnson L A 1978 J. Appl. Phys. 491769

Saegusa N and Morrish A H 1982 Phys. Rev. B26 10 